\title{
ON THE RELIABILITY PROBLEMS OF STRUCTURES SUBJECTED TO DYNAMICAL LOADS
}

\author{
Nguyen Van Pho, Le Ngoc Thach, Chu Thanh Binh \\ Hanoi University of Civil Engineering
}

\begin{abstract}
Mechanically, the assessment of the structure's safety relates to three aspects: strenght, stability and oscillation. In the oscillation problems of structures, the safety conditions are conditions of frequency, amplitude, resonance, maximum displacement, etc..

In case that the structure itself contains random parameters and subjects to external loads that are also the random parameters (or random processes), the assessment of the structure's safety according to the deterministic inequalities of structural mechanics will be insignificant. Therefore, this should be assessed according to the probabilistic point of view, namely, according to the reliability.

The determination of reliability of the oscillation problems of structures encounters many difficulties because the outputs of the structural analysis problem are the random processes (or the random field). Meanwhile, up to now, the determination of a probability according to which a random process will belong to a given domain by mathematical method has not been sufficiently studied yet.

In this paper, the authors, originating from a general definition on the reliability of a system by V. V. Bolotin, assess the reliability of oscillating structure, by determining the upper and the lower bounds of the reliability.

The upper and the lower bounds of the reliability are recommended to be determined by determining the probability depending on only an inequality instead of determining the it depending on a system of inequalities. Thus, the determination is very favourable.
\end{abstract}

\section{INTRODUCTION}

Reliability is the most important parameter for assessment of the structure's safety.

Mechanically, the structure's safety relates to three main aspects of structural mechanics: strenght, stability and oscillation.

The above three aspects are expressed in design standards as limit states [1].

In static problems, the method for determining the reliability according to the conditions of strenght and stability has been relatively-fully studied $[2,3,4,5,6, \ldots]$.

In the dynamical problems of structures, the inertia force and time $t$, are involved, the problem becomes much more complicated.

In the static problems we are interested in only the reliable probability of random variables, in the dynamic problem, we do have to be interested the probability of the exceedings of the stochastic processes $[7,8,9,10, \ldots]$.

Therefore, to talk about the problem of reliability of the oscillating structure, we can say that there are many problems left undone. 


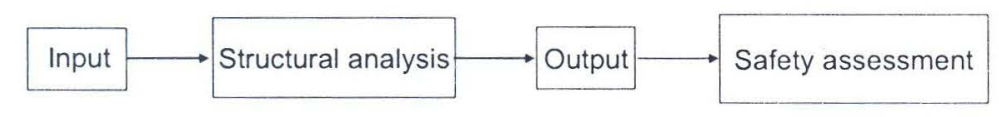

Fig. 1. The diagram of safety assessment of structures

As we all know, the analyzing problem of reliability of structures is carried out according to the following diagram [11]:

Input: deterministic parameters, random or fuzzy processes;

Structural analysis: familiar methods of dynamics of deterministic structures $[12,13]$, methods of random oscillation $[14,15,16, \ldots]$ and structural fuzzy analyzing methods $[17,18]$ may be used;

Output: random variables, random process, random field or fuzzy field;

Safety assessment: calculation of the reliability according to structural safety criterion.

When analyzing the reliability according to the diagram in Fig. 1, we face two main difficulties:

1. Solving the random oscillation problem of structures

The structure is a system of parameter distribution (innumerable degree of freedom), subjected to the action by stationary or nostationary random processes. Oscillation equation is a differential equation. Up to now, there have not been any effectively solving methods for these equations yet and they are often solved in specific cases.

2. Calculation of reliable probability:

Determination of a probability according to which a random process will belong to a given domain (zone) is a difficult problem. Up to now, mathematical achievements can only allow us to determine the probability according to which a random process exceeds a given threshold $[7,8,9,10]$, that is to say, the probability depends on an inequality only. Meanwhile, the reliable probability of structures, in fact, depends on a system of inequalities.

For the above mentioned reasons, to determine the reliability of a structure when it is oscillated, we have to simplify the problem and to find the approximate solution.

To improve the applicable ability of the solution of the above problem, we should appropriately use some assumptions or results obtained from the calculation of oscillation of deterministic structures.

In the specific oscillation problem, where there are only geometrical and physical parameters of the oscillation system, in many case this problem can be solved as a problem of reliability with random variables( free osillation).

In the forced oscillation problem, the output is generally random processes or, more exact, it is random field (because it depends on space parameters) $[8,9,10]$. Therefore, reliability problem is the problem of determining the probability of a random process belonging to a certain zone of the multi-direction space.

In this paper, the authors use the general definition of system's reliability by $\mathrm{V}$. V. Bolotin [8] to bring forward the way to assess the reliability of an oscillation system by finding out the upper and lower bounds of the safety probability. 
By finding out the upper and lower bounds of the safety probability, mathematically the problem of determining a probability that satisfies a system of inequalities changes into the problem of determining a probability that satisfies an inequality only.

By approximate calculation according to discrete time, the problem of reliability of a random process changes into the problem of reliability of a random variable.

To illustrate the proposed method, some problems of reliability of one or more degree of freedom structures are considered.

\section{THE LOWER AND UPPER BOUNDS OF RELIABILITY IN THE OSCILLATION PROBLEM}

\subsection{General definition of reliability}

In [8], V. V. Bolotin has defined that reliability of an oscillation system is a probability that satisfies, simultaneously, both the state equation and a system of inequalities ensuring the quality in space and time.

Let's call $\vec{u}=\vec{u}(\vec{x}, t)$ is the state vector of the oscillation system, where $\vec{x}=\left\{x_{1}, x_{2}, x_{3}\right\}^{T}$ is space variables and $t$ is the time;

Let's call $\vec{q}=\vec{q}(\vec{x}, t)$ is the vector of external loads, it may be either random process or random field.

$$
\operatorname{Lu}(\vec{x}, t)=\vec{q}(\vec{x}, t)
$$

is the state equation, where $L$ is the differential operator or algebraic operator;

Let's call $\vec{v}(\vec{x}, t)=\left\{v_{i}\right\}$ is the vector of quality,

$$
M \vec{u}(\vec{x}, t)=\vec{v}(\vec{x}, t)
$$

is the transform of state variables $\vec{u}(\vec{x}, t)$ to quality variables $\vec{v}(\vec{x}, t)$ and $M$ is the transform operator.

To ensure the quality (or to ensure the safety) then

$$
f(\vec{v}) \in \Omega_{0}
$$

where $\Omega_{0}$ is the quality zone and $f(\vec{v})$ is the quality function.

Let's call $\mathrm{V}$ is the zone the system occupies in the space $\vec{x}=\left\{x_{i}\right\}$.

The reliability of the system is the probability:

$$
P(t)=\operatorname{Pr} o .\left\{\begin{array}{l}
L \vec{u}=\vec{q}(\vec{x}, \tau) \\
M \vec{u}=\vec{v}(\vec{x}, \tau) \\
f(\vec{v}) \in \Omega_{0} \\
\forall \vec{x} \in V \\
\forall \tau \in[0, t]
\end{array}\right\} .
$$

In the oscillation problem, (2.1) is the oscillation equation.

If the state variable $\vec{u}(\vec{x}, t)$ is chosen as the displacement of the structure and the quality variable $\vec{v}(\vec{x}, t)$ is chosen as the stress, $\sigma_{i j}(\vec{x}, t)$ then the operator $\mathrm{M}$ is the product of the following two translation operators:

From $\vec{u}(\vec{x}, t)$, according to geometrical equations we can find deformations $\varepsilon_{i j}(\vec{x}, t)$;

From $\varepsilon_{i j}$, according to laws for materials (e.g. Hooke law) we can find $\sigma_{i j}(\vec{x}, t)$.

The condition (2.3) is condition on frequency, amplitude, resonance and maximum displacement, etc ... 
The condition $\forall \tau \in[0, t]$ is understood that the probability is calculated simultaneously with all values of $t$ in the range of $[0, t]$.

The determination of $P(t)$ according to (2.4) is very difficult. For that reason the probability can only be determined in a specific cases.

In the quasi-static problems, based on the technical significance of parameters (corrosion, degradation of materials with time,..) we can determine the variable direction of $P(t)$.

For example, with regard to steel corroded that results in reduction of the reliability with time, variable direction of $P(t)$ shall be as that shown in Fig. 2; For reinforced concrete structures, in the first 50 years, the reliability is almost not changed. After that, due to creep and other degradation reasons, the reliability is gradually reduced as shown in Fig. 3.

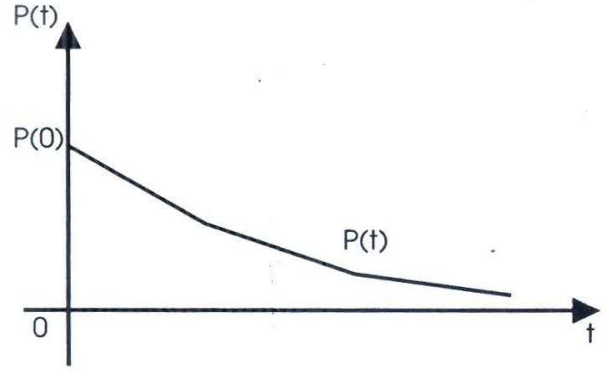

Fig. 2.

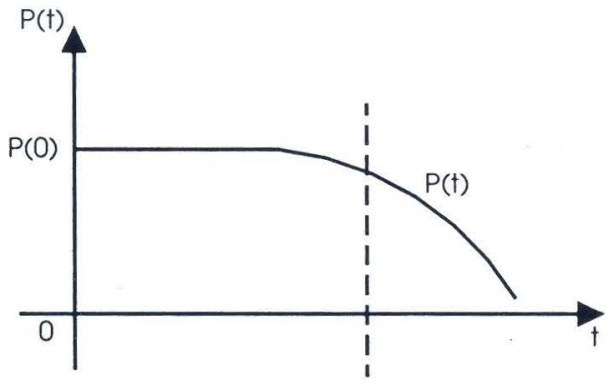

Fig. 3

In the dynamical problems, the probability $P(t)$ may be changed arbitrarily. For example, in the resonance region, $P(t)$ is low (see Fig. 4). Over the transition process of the oscillation, the probability changes insignificantly because the oscillation is stationary (see Fig. 5).

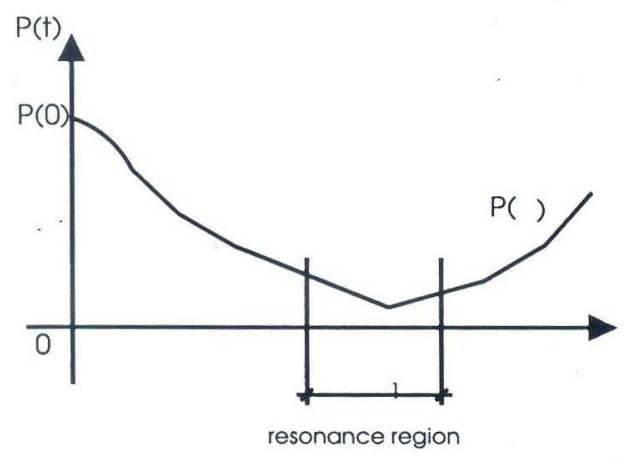

Fig. 4 .

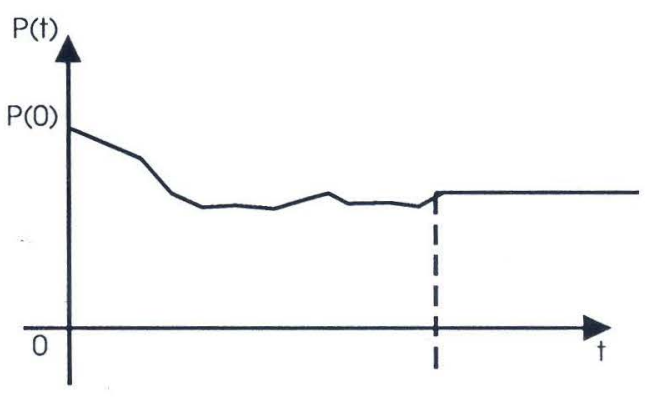

Fig. 5

In [6], we have proposed the way to determine the lower and upper bounds of reliability in the static problem (without the parameter of time). Hereinafter, we would like to expand the result obtained in [6] for oscillation problems. 


\subsection{The lower and upper bounds of $P(t)$}

Assumed that we have found out the solution for the state equation (2.2), carry out the alteration of (2.2) and apply the obtained result to (2.3) then we can have a probability the depends on a system of inequalities as follows:

$$
P(t)=\text { Pro. }\left\{\begin{array}{l}
f_{i}(\vec{x}, \tau) \leq 0 \\
\forall \vec{x} \in V \\
\forall \tau \in[0, t] \\
i=1,2, \ldots, m
\end{array}\right\} .
$$

To determine the approximate value of $P(t)$, we should be discretization according to space and time variables. For example, with regard to $t$, we examine at $\mathrm{n}+1$ discrete values $\left\{t_{k}\right\}=\left\{t_{0}=1, t_{1}, t_{2}, \ldots, t_{n}=n\right\}$ (see Fig. 6).

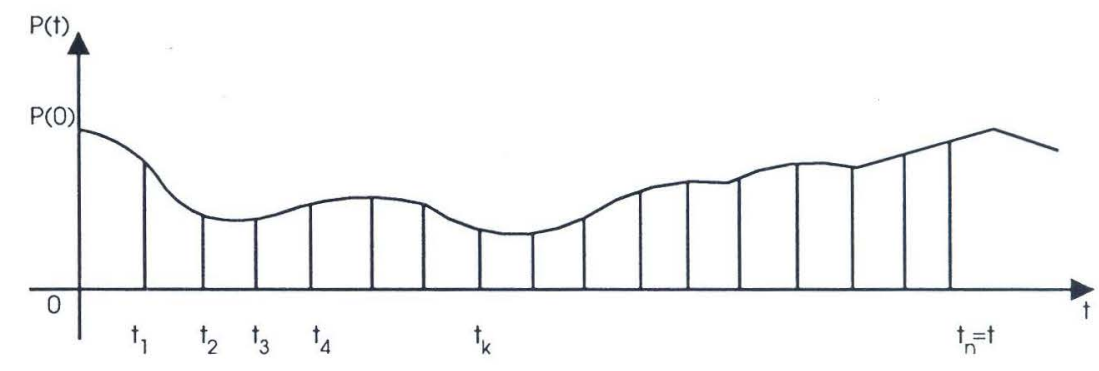

Fig. 6

Therefore, the probability (2.5) becomes the probability that depends, simultaneously, on a system of inequalities.

And now we calculate the probabilities that correspond to each equality. Let's give the probabilities the symbols $P_{i}, \mathrm{i}=1,2, \ldots$.

The calculation of probabilities that depend on only one equality can be easily carried out according to the proposed methods in $[2,3,4, \ldots]$.

Similar to [6], we are able to prove that the upper bound of $\mathrm{P}(\mathrm{t})$ shall be:

$$
P^{+}(t)=\max _{\{i\}} P_{i}
$$

and the lower bound of $P(t)$ shall be:

$$
P^{-}(t)=\min _{\{i\}} P_{i}
$$

This means:

$$
P^{-}(t) \leq P(t) \leq P^{+}(t)
$$

While it is simple to calculate $P^{+}(t)$ and $P^{-}(t)$, the quantity of calculation may be large when the number of inequality in (2.5) is large. This difficulty can be overcome for the time being, thanks to the personal computer.

Values of $P^{+}$and $P^{-}$have a very obvious technical significance:

When $P^{-}$is sufficient big, we can conclude that the structure is safe enough;

When $P^{-}$is small, it means that the reliability of some structural elements is small and then, we have to amend the design to increase the reliability of the weak structural elements; 
With similar doing way and with the re-examination by calculating, we can gradually obtain a structure with a "uniform reliability", that is the optimized structure.

With regard to existing structures, when the value of $P^{-}$of a structural element(s) is small, the element(s) shall be strengthened in order to increase the reliability of the whole structure.

In a structure, due to the architectural and aesthetic requirements, some structural elements may have a very high reliability $(\approx 1)$. It is a common situation if we cannot reduce the reliability of these elements. However, it is not permitted if $P^{-}$is too low.

Notes:

1) When examining the random processes, it is not allowed to specifically examine them at each discrete time because value of random processes at different times relates to each other with a correlation function, value of the correlation function represents the dynamical nature (characteristic) of the oscillation process.

In the above arguments, though the random processes is calculated according to the discrete time, the dynamical nature (characteristic) of the oscillation process is still maintained and this is shown in the determining the solution of the random oscillation equation (2.1) and the way to take min and max values (2.6) and (2.7).

2) During the oscillation process of the structure, generally the oscillation characteristics depend on the space variable $\vec{x}$ and time variable t. To simplify the process and also due to the importance of the time variable, we have shown clearly the discretization of the time variable rather than the discrete of the space variable. The space variable shall be discreted according to the net in finite element method (FEM);

3) There have been many research works on assessment of the reliability of a system by determining its lower and upper bounds $[2,3,4,6,19 \ldots]$. In these works, the authors have considered only some specific cases and the role of structural elements in the structure (serial, parallel or hybrid/combined) are based to propose the expression for the assessment.

In this paper we have relied upon the mathematical expressions only (i, e. the inequalities contained in the expression of reliability) to assess the structure's safety. Doing like this will facilitate the formulation of the problem as well as the calculation for solving the formulated problem.

\section{OSCILLATION OF ONE-DEGREE-OF-FREEDOM SYSTEM, RESONANCE REGION AND RELIABILITY}

\subsection{The randomness of parameters}

The randomness here is understood as the random deviations around the mean value that are expressed by the standard deviations. The deviations are ignored in the deterministic oscillation problem.

Without leaving the general characteristic, to simplify the problem, we should study the problem of one-degree-of-freedom system [12].

Let's study the oscillation of the mass $M$ placed on a beam of insignificant mass (the mass can be ignored) as shown in the Fig. 7a.

When subjected by a excitation force $P(t)$, the mass $M$ will oscillate around the balance position, $y=0$. At any time $t$, the location of mass $M$ shall be determined by vertical displacement $y(t)$. 


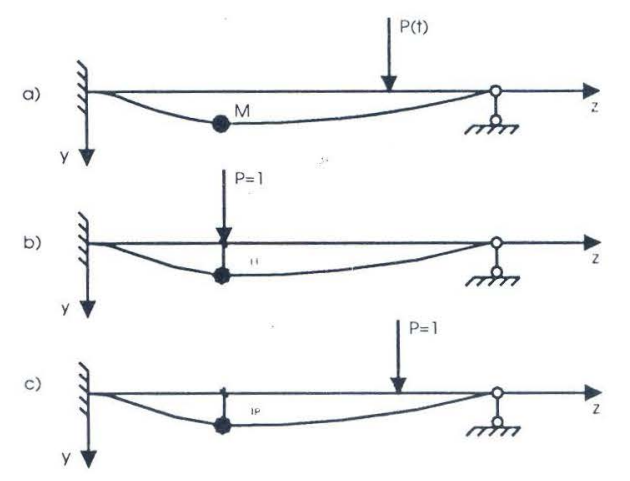

Fig. 7

The general differential equation of oscillation of the one-degree-of-freedom system shall be:

$$
\ddot{y}(t)+2 \alpha \dot{y}(t)+\omega^{2} y(t)=\omega^{2} \delta_{1 P} P(t)
$$

where:

$$
2 \alpha=\frac{\beta}{M}, \quad \omega^{2}=\frac{1}{M \delta_{11}}
$$

$\beta$ is the coefficient that takes into account the elastic-viscous property of the material, that is also called "resistance coefficient". The coefficient is involved in the internal friction of joints at the supports and that between structural elements of a structure, etc...

In (3.1), the model for elastic-viscous deformed materials assumed by Voigt is applied. According to this model, the resistance force is considered to be directly proportional to the displacement velocity.

$\delta_{11}$ is displacement in the moving direction, at the section where the mass $M$ is placed, caused by a force of $P=1$, statically acting on $M$ (Fig. 7b).

$\delta_{1 P}$ is displacement that is similar to $\delta_{11}$ but, it is caused by the force of $P=1$ placed at the same position of the simulating force (Fig. $7 \mathrm{c}$ ).

Thus, $\delta_{11}$ and $\delta_{1 P}$ depend on physical and geometrical properties of the beam.

The force $P(t)$ may either be deterministic or random.

It is obvious that the coefficients of (3.1) are random values, because they are either the experimental constants or functions of experimental constants obtained by processing the statistical data.

It is indeed true. To illustrate, let's study the determination of $\beta$ as follows as an example.

The resistance coefficient $\beta$ is selected for a free oscillation as follows [12].

Let's call the ratio: $\eta=\frac{y_{m}}{y_{m+1}}=e^{\alpha T_{1}}$ is the ratio between the two oscillation amplitudes that take place apart from each other by the period of $T_{1}$. Let's call $\chi=\ln \eta=\alpha T_{1}$ is the logarithm reduction of the oscillation. If the oscillation amplitudes (that take place apart from each other by the period of $T_{1}$ ) are measured with experiment, we can determine $\chi$ and then, from $\chi$, we can determine $\alpha$ and in the end, from $\alpha$ we can determine $; 3$ according to (3.2).

As a random value, $\beta$ is determined by its numerical characteristics (for example, the mean value and the standard deviation). 
In deterministic calculation, deviations around the average value are ignored and they are taken into account by permitting (allowing) design engineers to choose the value of $\alpha$ in a given range. Specific value of $\alpha$ shall be decided according to the experience of the designer.

The following table allows us to choose $\alpha$ after $T_{1}$ is known.

\begin{tabular}{llc}
\hline $\mathrm{N}^{0}$. & Structural type & $\alpha T_{1}$ \\
\hline 1 & Steel structures & $(0.016: 008) .2 \pi \approx 0.1: 0.5$ \\
2 & Timber structures & $(0.005: 0.022) .2 \pi \approx 0.031: 0.15$ \\
3 & Reinforced concrete structures & $(0.016: 0.032): 2 \pi \approx 0.08: 0.2$ \\
4 & Reinforced concrete beams & $(0.017: 0.39) \approx 0.28$ \\
5 & Reinforced concrete frames & $(0.080: 0.16) \approx 0.12$ \\
6 & Steel bridges & $(0.01: 0.15) \approx 0.08$ \\
7 & Reinforced concrete bridges & 0.31 \\
\hline
\end{tabular}

However in many cases of calculating according to the average value, the ignorance of deviation is not be suitable. On the other hand, the selection of the constant by experience and subjectivism by the engineer shall not allow us to assess the error.

Therefore, nowadays it is specified to design building structures according to the probability [1], meaning that the deviation is taken into account when designing.

In some current building design standards, reliability specification is not clearly stated, however, when developing the new standards or improving the old ones, reliability theory was applied to adjust the design coefficients.

\subsection{Resonance region and the reliability problem}

Let's study a simple case: a forced oscillation in which a resistance force is taken into account, with a exitation force $P(t)=P \cdot \sin \theta t$.

After the transition process, the oscillation become stable. The oscillation equation is expressed as follows:

$$
y(t)=\frac{y_{s}^{*}}{\sqrt{\left(1-\frac{\theta^{2}}{\omega^{2}}\right)^{2}+\gamma^{2} \frac{\theta^{2}}{\omega^{2}}}} \sin (\omega t-\varepsilon),
$$

where $y_{s}^{*}=\delta_{1 P} \cdot P$ is the static displacement caused by $P$ at the position placing the mass.

$$
\varepsilon=\operatorname{arctg} \frac{2 \alpha \theta}{\omega^{2}-\theta^{2}} \quad, \quad \gamma=\frac{2 \alpha}{\omega} ;
$$

It is clear that $y(t)$ is a random function of time (it is a random process).

Due to the requirements on either safety or serviceability, the oscillation amplitude is limited to a given range of values. For example:

$$
A \leq \frac{y_{s}^{*}}{\sqrt{\left(1-\frac{\theta^{2}}{\omega^{2}}\right)^{2}+\gamma^{2} \frac{\theta^{2}}{\omega^{2}}}} \leq B
$$

where $A$ and $B$ are the constants.

The inequalities (3.4) are the inequalities for the random values. Therefore, we can not replace random values in (3.4) with their respective mean values (according to the 
way applied to deterministic problems) to assess. Instead, we have to replace them with the probability that satisfies (3.4):

$$
\text { Prob. }\left\{A \leq \frac{y_{s}^{*}}{\sqrt{\left(1-\frac{\theta^{2}}{\omega^{2}}\right)^{2}+\gamma^{2} \frac{\theta^{2}}{\omega^{2}}}} \leq B\right\} \text {. }
$$

In the current deterministic calculation [12], within a level permissible approximation, the resonance condition is determined as:

$$
\frac{3}{4} \leq \frac{\theta}{\omega} \leq \frac{5}{4}
$$

To assess the accuracy of the resonance condition (3.6), we have to calculate:

$$
\text { Prob. }\left(\frac{3}{4} \leq \frac{\theta}{\omega} \leq \frac{5}{4}\right)=P_{f} .
$$

The probability (3.7) is the probability by which the oscillation is in the resonance region. The probability by which the oscillation is not in the resonance region (resonance safety probability) will be: $P_{s}=1-P_{f}$.

With different bounds' values of the resonance region, we have different values of $P_{s}$.

Notes: Because there is a factor (multiplier) of $\sin (\theta t-\varepsilon)$ in the expression (3.3) of $\mathrm{y}(\mathrm{t})$ and $\max _{\{t\}}(\sin (\theta t-\varepsilon))=1$, to simplify (3.6) and (3.7), we ignore the variable $\mathrm{t}$. If doing so, we can only find out the lower bound of (2.1).

Example: A motor with a volume of $Q$ is placed in the middle of a simple I-shaped beam whose $N^{0}$. is 30. Its length is $l$. The rotary speed of the motor is $n(\mathrm{rpm})$. Due to the unevenly distributed mass, an inertia force $P(t)=P_{0}$. $\sin \theta t$ shall be created when the motor is rotating. The beam volume per unit of length is $q$ (see Fig. 8).

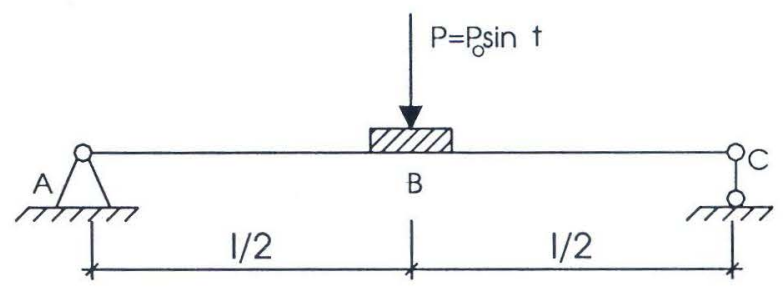

Fig. 8

$$
y_{s}^{*}=\frac{\left(Q+\frac{17}{35} q l\right)}{48 E I} l^{3}, \quad \omega=\sqrt{\frac{48 g E I}{\left(Q+\frac{17}{35} q l\right) l^{3}}}, \quad \theta=\frac{2 \pi n}{60} .
$$

Dynamic coefficient:

$$
\begin{aligned}
k_{d} & =\frac{1}{\sqrt{\left(1-\frac{\theta^{2}}{\omega^{2}}\right)^{2}+\frac{4 \alpha^{2} \theta^{2}}{\omega^{4}}}}, \\
\sigma_{\max } & =\frac{1}{8 w_{x}}\left(2 Q+q l+2 k_{d} P_{0}\right),
\end{aligned}
$$




$$
y_{\max }=\frac{l^{3}}{384 E I}\left(8 Q+5 q l+8 k_{d} P_{0}\right) .
$$

Probability of safety according to the durability condition will be:

$$
P_{s}^{(r)}=\operatorname{Prob} .\left(\sigma_{\max } \leq \sigma_{0}\right) .
$$

Probability of safety according to the rigidity condition will be:

$$
P_{s}^{(r)}=\operatorname{Pro} .\left(y_{\max } \leq[f]\right),
$$

With the following data:

$$
\begin{array}{lll}
\mu_{l}=400(\mathrm{~cm}), & \sigma_{l}=8(\mathrm{~cm}), & \mu_{w}=472\left(\mathrm{~cm}^{3}\right), \\
\sigma_{l}=9.44\left(\mathrm{~cm}^{3}\right), & \mu_{I}=7080\left(\mathrm{~cm}^{4}\right), & \mu_{\sigma_{0}}=20\left(\mathrm{kN} / \mathrm{cm}^{2}\right), \\
\sigma_{I}=141.6\left(\mathrm{~cm}^{4}\right), & \mu_{Q}=60(\mathrm{kN}), & \sigma_{Q}=10(\mathrm{kN}), \\
\mu_{P_{0}}=6(\mathrm{kN}), & \sigma_{P_{0}}=0.6(\mathrm{kN}), & \left.\sigma_{\sigma_{0}}=1 \mathrm{kN} / \mathrm{cm}^{2}\right), \\
\mu_{q}=0.00365(\mathrm{kN} / \mathrm{cm}), & \sigma_{q}=0.000365(\mathrm{kN} / \mathrm{cm}), & \mu_{n}=320(\mathrm{vong} / \mathrm{phut}), \\
\sigma_{n}=16(\mathrm{vong} / \mathrm{phut}), & \mu_{g}=981\left(\mathrm{~cm} / \mathrm{s}^{2}\right), & \sigma_{g}=49.05\left(\mathrm{~cm} / \mathrm{s}^{2}\right), \\
\mu_{k_{d}}=2.65, & \sigma_{g}=0.3, & \mu_{E}=2.1 x 10^{4}\left(\mathrm{kN} / \mathrm{cm}^{2}\right), \\
\sigma_{E}=0.105 x 10^{4}\left(\mathrm{kN} / \mathrm{cm}^{2}\right), & \mu_{[f]}=\frac{l}{400}, & \sigma_{[f]}=0.05 x \mu_{[f]},
\end{array}
$$

We have: $P_{s}^{(s)}=0.9839 ; P_{s}^{(r)}=0.99949$;

And now, we calculate the probability by which, the resonance takes place.

Supposing that the resonance region is (3.6), with the above data, we have: $P_{s}=$ 0.2776 so $P_{f}=1-P_{s}=0.7224$.

If we extend the resonance region, the probability by which, the resonance takes place will increase. For example, if we replace the condition in (3.6) with the following condition: $\frac{2.5}{4} \leq \frac{\Omega}{\omega} \leq \frac{5.5}{4}$ then $P_{s}=0.278$ so $P_{f}=1-P_{s}=0.722$.

If we choose a small expectation for $\frac{\Omega}{\omega}$ then the resonance region will be reduced and Ps will increase.

\section{OSCILLATION OF LIMITED DEGREE-OF-FREEDOM SYSTEMS}

In case that the system has $n$ degree-of-freedom, in general we have $n$ individual frequencies and $n$ types of correspondingly individual oscillations.

In this case, safety conditions are the conditions imposing on individual frequencies and the types of correspondingly individual oscillations.

In case of parameter-distributed systems, i.e. the systems with numberless degreeof-freedom, due to the discreting with time and space and, due to the concentration of mass, numberless degree-of-freedom systems becomes limited degree-of-freedom systems. If we simplify the problem according to this way, error is sure to be involved. However at present, there are quite a few research works solving the problem of model's error.

In technical calculation, another approximate method is being used as follows. Based on the solution for the deterministic case of the problem (supposing that we have obtained the solution for the deterministic oscillation problem), we know the dangerous position and time of the system. By this, the factors of time and space are excluded from the problem and then, we can have a problem with least degree-of-freedom as possible. 
Example: Determine individual frequencies and the types of correspondingly individual oscillations of a cantilever beam that has two degrees-of-freedom (see Fig. 9).

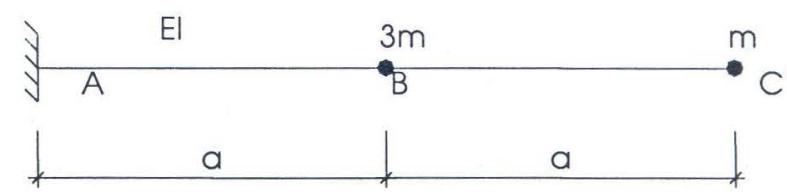

Fig. 9

Give values to necessary variables [12], we have the individual frequencies:

$$
\omega_{1}=0.5345 \sqrt{\frac{E I}{M a^{3}}} ; \quad \omega_{2}=2.50 \sqrt{\frac{E I}{M a^{3}}}
$$

and the main types of correspondingly individual oscillations:

$$
\begin{aligned}
& y_{11}(t)=y_{11} \cdot a_{1} \cdot \sin \left(\omega_{1} t+\varphi_{1}\right)=a_{1} \cdot \sin \left(\omega_{1} t+\varphi_{1}\right) . \\
& y_{21}(t)=y_{21} \cdot a_{1} \cdot \sin \left(\omega_{1} t+\varphi_{1}\right)=3 \cdot a_{1} \cdot \sin \left(\omega_{1} t+\varphi_{1}\right) .
\end{aligned}
$$

The values $a_{1}, \varphi_{1}, a_{2}, \varphi_{2}$ are determined from the initial conditions of the oscillation, i.e, at the time of $t=0$.

$$
\left\{\begin{array}{l}
a_{1} \sin \varphi_{1}+a_{2} \sin \varphi_{2}=y_{1}(0) \\
\omega_{1} a_{1} \cos \varphi_{1}+\omega_{2} a_{2} \cos \varphi_{2}=v_{1}(0) \\
3 a_{1} \sin \varphi_{1}+a_{2} \sin \varphi_{2}=y_{2}(0) \\
3 \omega_{1} a_{1} \cos \varphi_{1}-\omega_{2} a_{2} \cos \varphi_{2}=v_{2}(0)
\end{array}\right.
$$

Let's choose: $x_{1}=a_{1}, x_{2}=\sin \varphi_{1}, x_{3}=a_{2}, x_{4}=\sin \varphi_{2}, x_{5}=\cos \varphi_{1}, x_{6}=\cos \varphi_{2}$; the system (3.10) becomes a system of non-liner algebra equations as follows:

$$
\left\{\begin{array}{l}
x_{1} x_{2}+x_{3} x_{4}=y_{1}(0) \\
\omega_{1} x_{1} x_{5}+\omega_{2} x_{3} x_{6}=v_{1}(0) \\
3 x_{1} x_{2}+x_{3} x_{4}=y_{2}(0) \\
3 \omega_{1} x_{1} x_{5}-\omega_{2} x_{3} x_{6}=v_{2}(0) \\
x_{2}^{2}+x_{5}^{2}=1 \\
x_{6}^{2}+x_{4}^{2}=1
\end{array}\right.
$$

The system of non-liner equations (3.11) consists of 6 equations with 6 unknowns.

By solving (3.11) we can find out: $x_{i}=X_{i}\left(\omega_{1}, \omega_{2}, y_{1}(0), y_{2}(0), v_{1}(0), v_{2}(0)\right)(i=$ $1,2, \ldots, 6)$

With a way similar to that for the above mentioned part, we can determine the reliability of the system according to the resonance and displacement conditions of the beam with an attention that the system has two degrees-of-freedom. In the ordinary structures, only the first basic frequency is taken inrto account. However in the tall buildings and soft structures subject to wind load and seismic load, it is specified that higher frequencies be taken into account. 


\section{CONCLUSION}

Up to now, the reliability in the problem of structures' oscillation has not been much researched yet. The opinions shown in this paper are the initial results;

The current methods for assessing the safety of the structure when it is oscillating often use approximate assumptions to simplify the problem. Therefore, the error is not yet assessed. On the other hand, for some particularly important structures or structures that are highly-sensitive to excitation force, it is required to assess the error. Therefore, the view point of probability has to be used to solve the problem in order to expect a good result.

In this paper, it is supposed that the state equation (2.1) is solvable. What should we do if solutions of the equation (2.1) are the numerical solutions.

In analyzing structures, for almost all the equations, we can only determine the numerical solutions rather than the analytic solutions(mathematical expressions). Due to the discreting according to the time, we can change the calculation according to process into the calculation according to random values, i.e. we have changed the dynamical problem into the static one of reliability theory. In the static problem of reliability, we are allowed to use the approximate solutions $[2,3,4,6, \ldots]$.

Acknowledgement. This paper is completed with the financial assistance from the " Basic research programme in natural science fields (Mechanical discipline)". The Authors would like to thank to Ministry of Science and Technology of VietNam and the chairing board of the Basic research programme in Natural science fields.

\section{REFERENCES}

1. International Standart: ISO 2394-1998, General principles on Reliability for structures.

2. Pall Thoft-Christensen, Yoshisada Murotsu, Aplication of Structural Systems Reliability Theory, Springer-Verlag 1986.

3. O.Ditlevsen, H.O Madsen, Structural Reliability Methods, Wiley-1996.

4. Achintya Haldar, Probability, Reliability and Staticstical Methods in Engineering Design, John Wiley and sons, New York, Toronto, Singapore, 2000.

5. Nguyen Van Pho, A new method for the deternation of the reliability index of distributed parameter systems, Vietnam Journal of Mechanics 4 (2003) 186-192.

6. Nguyen Van Pho, Nguyen Xuan Chinh, Ta Thanh Van, A method for Reliability assessment of structures, Proceedings of the Eighth National Conference on Solid Mechanics Thai Nguyen 25-26 August, 2006, 618-627.

7. V. I. Tikhonov, Exceedings of Stochastic Processes, Moscow. Nauka-1970 (In Russian).

8. V. V. Bolotin, Methods of Probability Theory and Reliability Theory in the Calalution of Structures, Moscow-Stroizdat 1982 (In Russian).

9. E. C. Pereverzev, Stochastic Processes in Parameter Reliability Model, Kiev, Nauka dumka1987 (In Russian).

10. V. V. Bolotin, Statistical Methods in Structural Mechanic, Moscow-Stroizdat-1965 (In Russian).

11. Nguyen Van Pho, Le Ngoc Thach, Tran Van Lien, Diagnostic problems of structures in situation of fuzzy information, Proceedings of the eighth national conference on solid mechanics Thai Nguyen 25-26 August, 2006, 618-627 (In Vietnamese).

12. Nguyen Van Phuong, Structural Dynamics, Science and Technique Publishing House, Ha noi2005 (In Vietnamese). 
13. W. C. Hurty and M.F Rubinstein, Dynamics of Structures, Prentice-Hall Englewood Cliffs N. J. 1964.

14. Y. K. Lin, Probabilistic Theory of Structural Dynamics, McGrow-Hill. 1967.

15. V. P. Makeev, N. I. Grinkenko, Iu. C. Pavluk, Statistical Dynamic Problems of Elastic Structures, Moscow-Nauka-1984 (In Russian).

16. Bogdan skalmierski, Andzey Tylikowski, Stochastic Processes in Dynamics PWN-Polish, Scientific publishers, Warsszawa-1982.

17. Bernd Moller, Michael Beer, Fuzzy Randomness, Uncertainty in civil Engineering and computational mechanics, Springer 2004.

18. Nguyen Van Pho, Mechanics in the situation of fuzzy information, Proceedings of the Seventh National Conference on Solid Mechanics Do Son 27-28 August, 2004, 665-675 (In Vietnamese).

19. Chu Thanh Binh, Le Ngoc Thach, Some reliability problems of the bending beam, Proceedings of the Eighth National Conference on Solid Mechanics Thai Nguyen 25-26 August, 2006, 59-67 (In Vietnamese)

20. Nguyen Van Pho, Le Ngoc Thach, Method of linearization for reliability problems, Vietnam Journal of Mechanics 3 (1993) 45-51.

Received June 15, $200 \%$.

\section{VỀ CÁC BÀI TOÁN DỘ TIN CẬY CƯA KẾT CẤU CHỊU TẢI TRỌNG DộNG LựC}

Về mặt cơ học, việc đánh giá an toàn của công trình có liên quan đến 3 lĩnh vực: độ bền, ỡn định và dao động công trình. Trong bài toán dao động công trình thì điều kiện an toàn là các điều kiện về tần số, biên độ, cộng hưởng, chuyển vị cực đại v.v.. Trong trường hợp bản thân công trình chứa các tham số ngẫu nhiên và chịu tác động của tải trọng ngoài là các đại lượng ngã̃u nhiên (hay quá trình ngẫu nhiên) thì việc đánh giá theo các bất đẳng thức tất định của cơ học kết cấu là không có nghĩa. Do đó, người ta phải đánh giá theo quan điểm xác suất, nghĩa là phải tính độ tin cậy.

Tìm độ tin cậy của bài toán dao động công trình gặp nhiều khó khăn, vì đầu ra của bài toán phân tích kết cấu là các quá trình ngẫu nhiên (hay trường ngẫu nhiên). Trong khi đó, cho đến nay các thành tựu của toán học về tính xác suất để một quá trình ngẫu nhiên nằm trong một miền nào đó, chưa được nghiên cứu đầy đủ.

Trong bài này, các tác giả xuất phát từ định nghĩa tổng quát về độ tin cậy của hệ thống của V. V. Bolotin, để đánh giá độ tin cậy của công trình dao động bằng cách tìm cận trên và dưới của độ tin cậy.

Đề xuất cách tính cận trên và cận dưới của độ tin cậy bằng cách chuyển việc tính xác suất phụ thuộc một hệ bất đẳng thức về tính xác suất chỉ phụ thuộc một bất đẳng thức. Nhờ vậy, rất thuận lợi cho việc tính toán. 